\title{
Neurodevelopmental outcomes and comorbidities of children with congenital muscular torticollis: evaluation using the National Health Screening Program for Infants and Children database
}

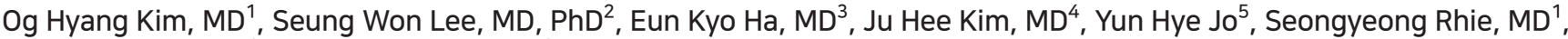 \\ Man Yong Han, $\mathrm{MD}^{1}$, Kyu Young Chae, $\mathrm{MD}^{1}$ \\ 1 Department of Pediatrics, CHA Bundang Medical Center, CHA University School of Medicine, Seongnam, Korea; ${ }^{2}$ Department of Data Science, Sejong University \\ College of Software Convergence, Seoul, Korea; ${ }^{3}$ Department of Pediatrics, Kangnam Sacred Heart Hospital, Hallym University Medical Center, Seoul, Korea; \\ ${ }^{4}$ Department of Pediatrics, Kangdong Sacred Heart Hospital, Seoul, Korea; ${ }^{5}$ Department of Global Economic, Gachon University, Seongnam, Korea
}

Background: Congenital muscular torticollis (CMT) is a common musculoskeletal disorder occurring at birth or in infancy. Purpose: This study aimed to investigate the risk of comorbidities in CMT and explore the differences in neurodevelopmental outcomes between children who received physical therapy and those who did not.

Methods: Children with CMT born in 2008 and 2009 in Korea were included. CMT was defined as a primary diagnosis of congenital deformity of sternocleidomastoid muscle according to the International Classification of Diseases 10th revision. Here we investigated the associated neurological/ musculoskeletal comorbidities of children with CMT versus those of the general population. We divided those with torticollis into treatment and nontreatment groups and assessed their developmental outcomes within both groups of children using the Korean-Ages and Stages Questionnaire (K-ASQ).

Results: Of the 917,707 children, $0.2 \%(n=1,719)$ were diagnosed with CMT. In children with torticollis, the prevalence of congenital hip deformities significantly increased to $4.5 \%$ $(n=78)$. The prevalence of congenital head/spine deformities and other congenital malformations of the skull and facial bones increased to $2.6 \%(n=44)$, while the prevalence of congenital foot deformities was $2.4 \%(n=42)$. The risk ratio (RR) for delayed development based on the K-ASQ was higher for the total assessment (adjusted RR $=0.97 ; 95 \%$ confidence interval, 0.93-0.99) in the CMT patients without physical therapy than in those with therapy. There was no significant intergroup difference in the assessment of each developmental area.

Conclusion: The prevalence of comorbid musculoskeletal deformities was higher in children with CMT than in the control group. The risk of developmental delay was higher in children who did not receive physical therapy than in those who did.
Key words: Congenital torticollis, Child, Comorbidity, Developmental disabilities

\section{Key message}

Question: What comorbidities are increased in children with congenital muscular torticollis (CMT)? Are there differences in the neurodevelopmental outcomes of children with CMT who received physical therapy versus those who did not?

Finding: The risk of congenital musculoskeletal deformities is increased in CMT. Children who did not receive physical therapy were at greater risk of neurodevelopmental delay.

Meaning: In CMT, musculoskeletal comorbidities should be identified and active early treatment provided.

\section{Introduction}

Torticollis, literally meaning "twisted neck," may present as a single disease. However, torticollis may be a clinical manifestation of various underlying conditions such as congenital disorders, trauma, inflammation, and neurological diseases.

Congenital muscular torticollis (CMT), the most common musculoskeletal disorder that occurs at birth or in infancy, is characterized by a contracted and shortened sternocleidomastoid muscle (SCM), which results in tilting of the head toward the affected muscle and rotation of the chin to the opposite side. ${ }^{1-3)} \mathrm{CMT}$ is presumed to occur as local fibrosis with a contracture accompanied by muscle-localized ischemia caused by compression, deformation, or stretching of the SCM. ${ }^{4}$

In children with CMT, the incidence of developmental dysplasia of the hip may increase due to intrauterine crowding, and positional comorbidities may occur due to the twisted posi-

\footnotetext{
Corresponding author: Kyu Young Chae, MD. Department of Pediatrics, CHA University School of Medicine, 59, Yatap-ro, Bundang-gu, Seongnam 13496, Korea 凶Email: danielchae21@gmail.com, https://orcid.org/0000-0003-3243-5853 


\section{Congenital Muscular Torticollis}

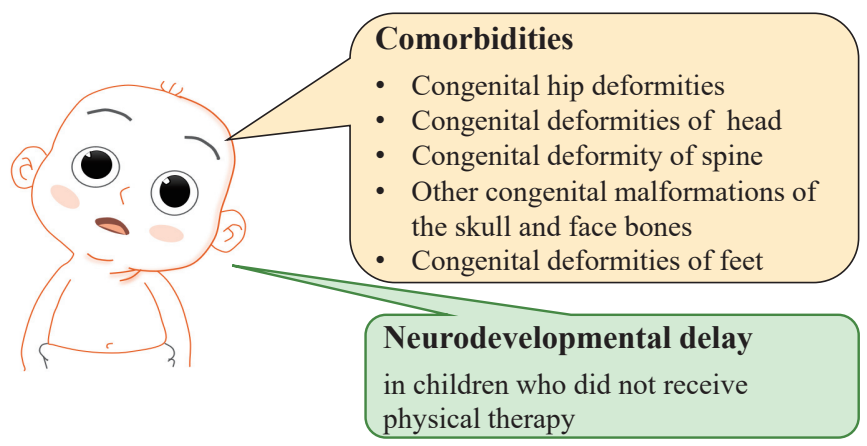

In children with CMT, musculoskeletal comorbidities should be identified and active early treatment should be performed to prevent neurodevelopmental delays.

\section{Graphical abstract}

tion. ${ }^{3,4)}$ Disorders associated with CMT include plagiocephaly, facial asymmetry, and positional musculoskeletal deformities such as cervical scoliosis, vertebral wedge degeneration, metatarsus adductus, and calcaneovalgus feet (talipes calcaneovalgus). Although there are several prior studies of comorbidities associated with CMT, they have not explored other comprehensive neuromuscular skeletal disorders and have been limited to a small scale. ${ }^{5-8)}$

If CMT is not treated early, it can affect the child's appearance, and cause visual impairment and neurodevelopmental delay. ${ }^{9-13)}$ Conservative treatment of CMT includes home stretching and Physical and Rehabilitation Medicine (PRM) intervention. Postural torticollis identified in an early phase can be rapidly resolved with positioning or simple home stretching, but most cases diagnosed after 3-6 months of age or severe type with SCM mass require long-term exercise therapy by PRM physician. ${ }^{14}$ Exercise therapy for CMT includes several components, such as manual stretching of SCM muscle, active rotation exercises, positioning and handling, and environmental adaptation, parent/ caregiver education. ${ }^{15)}$

There have been controversies over early treatment in children with CMT. Many studies' results indicated that early interventional treatment can reduce secondary damage or complications in CMT patients. ${ }^{4,14,16)}$ Other studies reported that most patients spontaneously resolved within the first year of birth. ${ }^{7,17)}$ To our knowledge, there has been no large-scale study that has investigated and shown the developmental outcomes of the treatment and nontreatment cases in CMT.

Accordingly, the aim of this study was to investigate the risk of more comprehensive comorbidities related to CMT using a population-based nationwide design. In addition, we attempted to explore the difference in neurodevelopmental outcomes between the children with CMT who received physical therapy and those who did not.

\section{Methods}

\section{Study design and setting}

This retrospective study was conducted on children diagnosed with CMT among 917,707 children born in Korea in 2008 ( $\mathrm{n}=$ 469,248) and $2009(n=448,459)$ and followed up through 2017. Of the 917,707 children, 10,000 who had never been diagnosed with CMT were randomly selected as the control group. We investigated the frequency of comorbid diseases commonly associated with CMT in each group, respectively. We divided children with CMT into treatment and nontreatment groups and compared neurodevelopmental outcomes. The analysis included children who had been screened by at least one National Health Screening Program for Infants and Children (NHSPIC) for children from ages 42 to 71 months (Fig. 1).

Data were collected from the National Health Insurance Service (NHIS) and the NHSPIC. The NHIS is a single insurer system that covers almost all of Korea's population and the NHSPIC is a child surveillance program consisting of 7 followup screening surveys for infants and children from the age of 4 to 71 months. The NHIS provides insurance benefits for medical

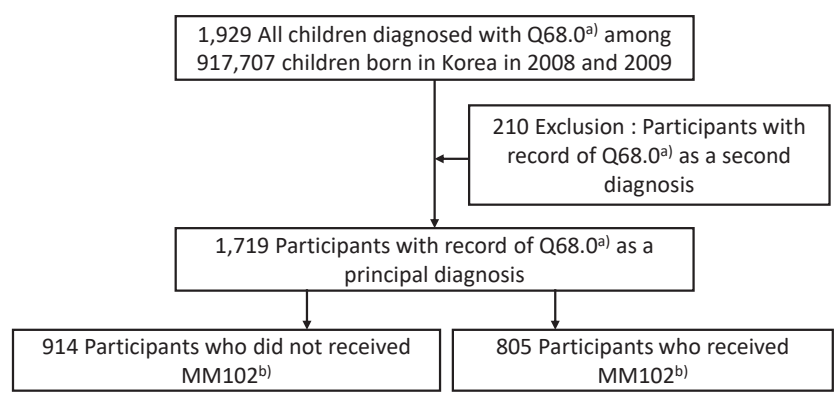

Fig. 1. Flow diagram of the study participant enrollment. ${ }^{a} \mathrm{Q} 68.0$ means congenital deformity of sternocleidomastoid muscle in the International

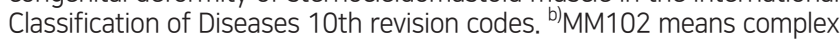
therapeutic exercise. 
services including injuries, childbirth, deaths, health promotions, rehabilitations, or preventions of diseases, and maintains national records for healthcare utilization, prescriptions, and health screening programs. The NHSPIC survey includes a general health questionnaire, developmental screening, an oral health questionnaire, oral examinations and guidance, anthropometric examinations, physical examinations, and anticipatory guidance. ${ }^{18)}$ Clinical information of children was collected from medical charts and recorded using the International Classification of Disease, 10 th version (ICD-10).

The protocol of this study was reviewed and approved by the Institutional Review Board of the Korea National Institute for Bioethics Policy (P01-201603-21-005) and conducted in accordance with the WMA Declaration of Helsinki: Ethical Principles for Medical Research Involving Human Subjects.

\section{Exposure definition}

CMT was defined as cases diagnosed with the primary diagnosis of "congenital deformity of sternocleidomastoid muscle"(Q68.0) according to the ICD-10 code. "Congenital deformity of sternocleidomastoid muscle" included "congenital (sternomastoid) torticollis," "contracture of sternocleidomastoid (muscle)" and "sternomastoid tumor (congenital)." Physical therapy for CMT was defined as receiving complex therapeutic exercise by physical therapists.

\section{Outcome definition}

The outcomes of this study were the comorbidities and neurodevelopmental outcomes in children with CMT. Comorbidities were defined as musculoskeletal diseases known as related disorders with CMT, and malformations of the nervous system. The prevalence of comorbidities was evaluated by ICD-10 diagnosis. These included congenital malformations of the nervous system (Q00-Q07), congenital malformations and deformations of the musculoskeletal system (Q65-Q79), and chromosomal abnormalities (Q90-Q99). Musculoskeletal deformations were classified into congenital hip deformities (Q65.*), congenital foot deformities (Q66.*), congenital musculoskeletal deformities of head (Q67.0-Q67.5), other congenital musculoskeletal deformities (68.* except 68.0), polydactyly, and syndactyly (Q69.*Q70.*), reduction of defect of upper lower, unspecified limb (Q71.*-Q74.*), other congenital malformations of skull and face bones (Q75.*), congenital malformations of spine (Q76.0 Q76.4), osteochondrodysplasia (Q77.*-Q78.*), and other congenital malformation of musculoskeletal system (Q79.*).

In this study, the neurodevelopmental outcomes were compared between the physical therapy and the nonphysical therapy groups using the developmental screening test. The developmental screening test is a major screening tool of the NHSPIC and is performed after 9 months of age (2nd through 7th screening surveys). From 2008 to 2013, the Korean Ages and Stages Questionnaire (K-ASQ) was used as the main developmental screening tool, and from 2014 onward, the Korean Developmental Screening Test for Infants and Children was mainly used. For the identicalness of research methods in this study, we only used the results of the 5 th to the 7 th developmental screening tests conducted through the K-ASQ. K- ASQ comprises 5 developmental areas (communication, gross motor skills, fine motor skills, problem-solving, and personal-social interrelation) that are assessed under 2 categories: "appropriate" and "need for follow-up." The total evaluation assesses the domains under 3 categories: "appropriate," "need for follow-up," and "recommendation for further evaluation." If a child is considered to have developmental delays based on further evaluation, the result is classified as "need for consistent management." If at least one of the responses to the "need for follow-up," "recommendation for further evaluation," and/or "need for consistent management" categories in the total evaluation is "yes," the result is classified as "need for evaluation." ${ }^{19)}$ There was some variability in the final study sample because there were children who failed to complete some areas of the K-ASQ.

\section{Covariate definition}

All covariates, such as sex, residence at birth, income quintile, type of milk feeding, and prematurity were collected from the NHIS database. The NHIS provides a public database of healthcare utilization, sociodemographic parameters, and outcomes of the NHSPIC. The economic status was divided into quintiles and determined by the amount of insurance copayment (1Q: poorest quintile, 5Q: richest quintile). Residence at birth was categorized as follows: Seoul, metropolitan areas (Busan, Daegu, Incheon, Gwangju, Daejeon, Ulsan), city (urban areas), and rural (noncity) areas.

\section{Data analysis}

The statistical comparison of the incidence of comorbidities in children with CMT was conducted by independent $t$ test. The difference between the treatment group and nontreatment group was significant if the $P$ value was less than 0.05 . We calculated risk ratio (RR) and $95 \%$ confidence interval (CI) using modified Poisson regression model to compare the difference in neurodevelopmental outcome between the 2 groups. We adjusted for RR considering sex, the residence of birth, household income, type of milk feeding, prematurity, and birth weight, as recorded in the database. All analyses were performed using SAS Enterprise Guide ver. 7.1 (SAS Institute Inc., Cary, NC, USA).

\section{Results}

\section{Incidence of CMT in Korea}

Of the 917,707 children, 1,719 (0.2\%) were diagnosed with CMT, and median age at diagnosis was 4 months of age (mean age \pm standard deviation [SD], $16.5 \pm 20.1$ months). Of children with CMT, 47\% $(\mathrm{n}=805)$ received physical therapy and 53\% $(\mathrm{n}=914)$ did not. The median age at onset of physical therapy in treatment group was 2 months of age (mean \pm SD, $3.2 \pm 2.5$ 
Table 1. Demographic characteristics of the study population

\begin{tabular}{|c|c|c|}
\hline Characteristic & $\begin{array}{l}\text { Congenital muscular } \\
\text { torticollis }(n=1,719)\end{array}$ & $\begin{array}{c}\text { General population } \\
(n=10,000)\end{array}$ \\
\hline \multicolumn{3}{|l|}{ Sex } \\
\hline Boy & $1,008(58.6)$ & $5,083(50.8)$ \\
\hline Girl & $711(41.4)$ & $4,917(49.2)$ \\
\hline \multicolumn{3}{|l|}{ Birth residence ${ }^{a}$} \\
\hline Seoul & $472(27.5)$ & $2,572(25.7)$ \\
\hline Metropolitan & $391(22.8)$ & $2,254(22.5)$ \\
\hline City & 599 (34.9) & $3,786(37.9)$ \\
\hline Rural & $241(14.0)$ & $1,283(12.8)$ \\
\hline Missing & $16(0.9)$ & $105(1.05)$ \\
\hline \multicolumn{3}{|l|}{ Income quintile ${ }^{b)}$} \\
\hline 1 (lowest) & $110(6.4)$ & $820(0.8)$ \\
\hline 2 & $221(12.9)$ & $1,457(14.6)$ \\
\hline 3 (middle) & $432(25.1)$ & $2,502(25.0)$ \\
\hline 4 & $610(35.5)$ & 3,131 (31.3) \\
\hline 5 (highest) & $295(17.2)$ & $1,702(17.00)$ \\
\hline Missing & $51(3.0)$ & 388 (3.9) \\
\hline \multicolumn{3}{|c|}{ Type of milk feeding ${ }^{c}$} \\
\hline Breast & $436(25.4)$ & $2,253(22.5)$ \\
\hline Bottle & $421(24.5)$ & $1,820(18.2)$ \\
\hline Mixed & $219(12.7)$ & $948(9.5)$ \\
\hline Special formula & $4(0.2)$ & $20(0.2)$ \\
\hline Missing & $6(0.4)$ & 4,959 (49.6) \\
\hline \multicolumn{3}{|l|}{ Birth year } \\
\hline 2008 & 857 (49.9) & $5,173(51.7)$ \\
\hline 2009 & $862(50.2)$ & 4827 (48.3) \\
\hline \multicolumn{3}{|l|}{ Prematurity } \\
\hline No & $1,537(89.4)$ & 8,695 (87.0) \\
\hline Yes & $113(6.6)$ & 504 (5.0) \\
\hline Missing & $69(4.0)$ & $801(8.0)$ \\
\hline
\end{tabular}

Values are presented as number (\%).

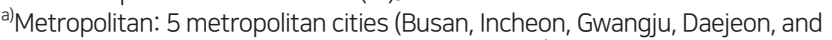

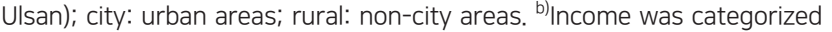
into quintiles of average neighborhood income on the index date. ${ }^{\mathrm{c}}$ Feeding with breast milk, animal milk, and/or formula milk. months). All children of the treatment group started therapy within 1 year of birth; 83.7\% (674 of 805) started within the first 6 months, and $16.3 \%$ (131 of 805 ) started at 6-12 months of age.

The general characteristics of children with CMT and controls are described in Table 1. The male to female ratio was $6: 4$ in the CMT group, and males were more prevalent. There was no difference in birth residence, income quintile, feeding type, and prematurity.

\section{Associated comorbidities in children with CMT}

In children with CMT, the incidence of congenital hip deformities was significantly increased to $4.5 \%(\mathrm{n}=78)$, and congenital deformities of the head including plagiocephaly and facial asymmetry, spinal deformities, other congenital malformations of the skull and face bones were increased to $2.6 \%(n=44)$. The incidence of congenital foot deformities including metatarsus adductus, and talipes calcaneovalgus was $2.4 \%(n=42)$. How ever, the incidence of neurologic deformities $(n=17)$ and chromosomal abnormalities $(\mathrm{n}=5)$ was less than $1 \%$ (Fig. 2, Table 2). Of the 1,719 children with CMT, $13.6 \%(n=233)$ had one or more congenital musculoskeletal deformities. One hundred sixty-five children had 1 comorbidity, 22 children had 2 , and 46 children had more than 3 comorbidities (Table 3 ).

\section{Neurodevelopmental outcome}

The RR for delayed development based on the results from the 5th-7th K-ASQ was higher for the total developmental area (adjusted RR $=0.97$; 95\% CI, 0.93-0.99) in the nontreatment group than in the treatment group. There was no significant difference between the 2 groups in the assessment of communication, gross motor, fine motor, problem-solving, or personal/ social developmental areas (Table 4).

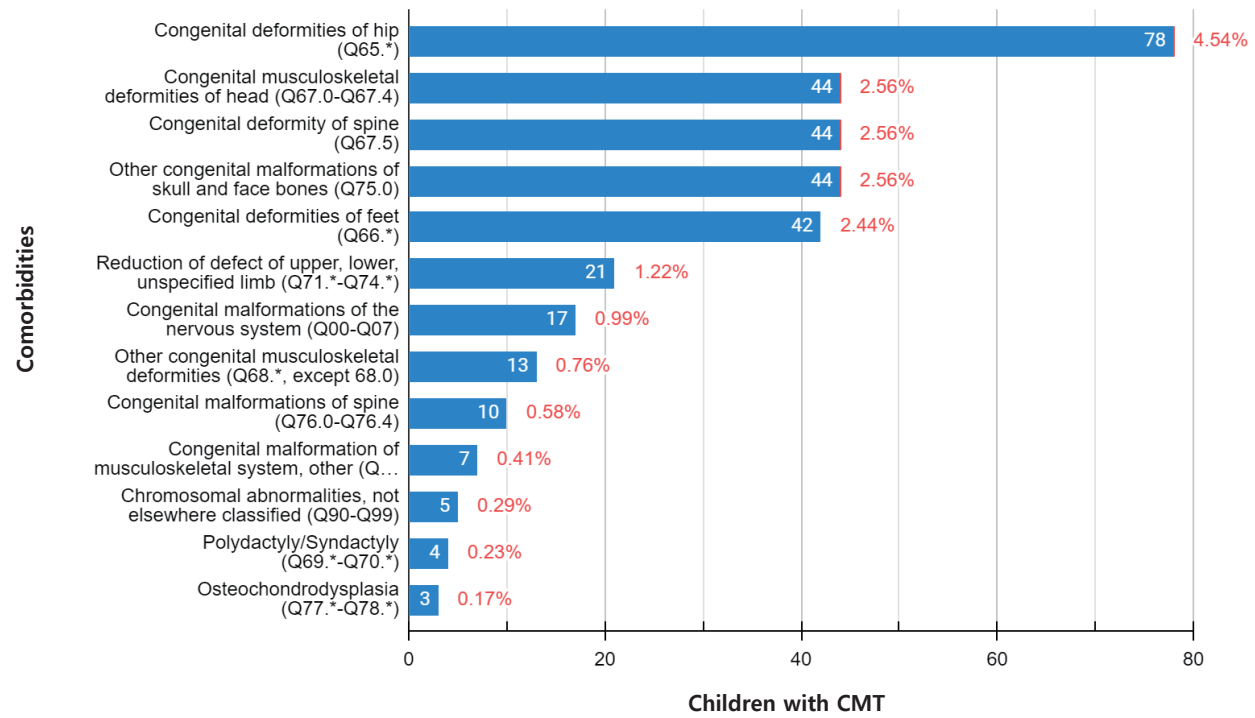

Fig. 2. Associated comorbidities of children with congenital muscular torticollis (CMT). 
Table 2. Associated comorbidities in children with congenital muscular torticollis

\begin{tabular}{|c|c|c|c|}
\hline Comorbidities $^{\mathrm{a})}$ & $\begin{array}{l}\text { Congenital muscular torticollis } \\
\qquad(n=1,719)\end{array}$ & $\begin{array}{l}\text { General population } \\
\quad(n=10,000)\end{array}$ & $P$ value \\
\hline Congenital malformations of the nervous system (Q00-Q07) & $17(1.0)$ & $0(0.0)$ & \\
\hline Congenital deformities of hip (Q65.*) & $78(4.5)$ & $2(0.02)$ & $<0.001$ \\
\hline Congenital deformities of feet (Q66.*) & $42(2.4)$ & $1(0.01)$ & 0.075 \\
\hline Congenital musculoskeletal deformities of head (Q67.0-Q67.4) & $44(2.6)$ & $1(0.01)$ & $<0.001$ \\
\hline Congenital deformity of spine (Q67.5) & $44(2.6)$ & $1(0.01)$ & $<0.001$ \\
\hline Other congenital musculoskeletal deformities (Q68.X, except 68.0) & $13(0.8)$ & $0(0)$ & \\
\hline Polydactyly/Syndactyly (Q69.*-Q70.*) & $4(0.2)$ & $0(0)$ & \\
\hline Reduction of defect of upper, lower, unspecified limb (Q71.*-Q74.*) & $21(1.2)$ & $0(0)$ & \\
\hline Other congenital malformations of skull and face bones (Q75.*) & $44(2.6)$ & $1(0.01)$ & $<0.001$ \\
\hline Congenital malformations of spine (Q76.0-Q76.4) & $10(0.6)$ & $0(0)$ & \\
\hline Osteochondrodysplasia (Q77.*-Q78.*) & $3(0.2)$ & $0(0)$ & \\
\hline Congenital malformation of musculoskeletal system, other (Q79.*) & $7(0.4)$ & $1(0.01)$ & $<0.001$ \\
\hline Chromosomal abnormalities, not elsewhere classified (Q90-Q99) & $5(0.3)$ & $0(0)$ & \\
\hline
\end{tabular}

Values are presented as number (\%).

Boldface indicates a statistically significant difference with $P<0.05$.

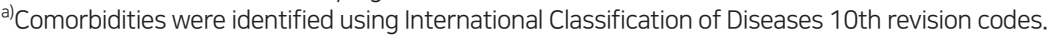

Table 3. Incidence of comorbidities in children with congenital muscular torticollis

\begin{tabular}{lc}
\hline No. of comorbidities & $\begin{array}{c}\text { Congenital muscular torticollis } \\
(\mathrm{n}=1,719)\end{array}$ \\
\hline 1 & $1,486(86.4)$ \\
2 & $165(9.6)$ \\
$\geq 3$ & $22(1.3)$ \\
\hline
\end{tabular}

Values are presented as number (\%).

\section{Discussion}

The prevalence of CMT in South Korea was 0.2\%. The risk of congenital hip deformities was the highest in CMT, and the risk of congenital deformities of the head, skull and facial bones, spine, and feet was also increased significantly. The risk of developmental delay was higher for children who did not receive physical therapy than those who did not.

The prevalence of CMT in South Korea was $0.2 \%$ in this study. Male to female ratio was 6:4. The incidence of CMT is reportedly $0.3 \%$ to $2 \%{ }^{4,17)}$; however, the incidence may be higher. Stellwagen et al. ${ }^{20)}$ reported that CMT affected 16\% $(\mathrm{N}=102)$ of newborns. The incidence of CMT in our study was lower than that reported in other studies. However, in our study, only cases with congenital deformity of the SCM were included. Since most of SCM tightness and postural torticollis without tumor were excluded by inclusion criteria, it is possible that our results are lower than reality. In this study, CMT was more common in males and was similar to previous studies. ${ }^{21,22)}$

Although the etiology of CMT remains unclear, birth trauma, ${ }^{23)}$ perinatal compartment syndrome, ${ }^{24)}$ and impairment of the developing SCM caused by intrauterine restrictions ${ }^{25-27)}$ are known causes. In addition, breech presentation, multiple pregnancies, primiparity, and dystocic labor are considered risk factors for $\mathrm{CMT}^{14)}{ }^{14}$ However, in our study, we could not investigate risk factors for CMT such as abnormal fetal positioning, continuous neck rotation in utero, and difficult delivery. In CMT, pathological features of the affected SCM include excessive endomysial and perimysial fibrosis, adipocyte hyperplasia, and muscle fiber atrophy. These features cause tightness of the involved SCM and limit cervical movement. An immunohistochemical study mentioned that deposition of type III collagen is a key factor in the SCM fibrosis that causes CMT, and that this hyperplasia is associated with accelerated apoptosis and overexpression of transforming growth factor-beta. ${ }^{28)}$

We analyzed the incidence of comorbidities in children diagnosed with CMT. In our study, the risk of congenital hip deformities was significantly increased. We also found the risk of congenital deformities of the head, skull and facial bones, spine, and feet to be increased. According to previous studies, 6\%-20\% of children with CMT have congenital musculoskeletal disorders including congenital hip dysplasia, craniofacial asymmetry, ${ }^{5)}$ cervical spine dysmorphism, ${ }^{6,29)}$ and plagiocephaly. ${ }^{7,30)}$ Hip dysplasia has been identified in 0\%-20\% children with $\mathrm{CMT}^{8}{ }^{8}$ In our study, hip dysplasia was found in $4.5 \%$ of the children with CMT. In addition, congenital deformities of the head including plagiocephaly and facial asymmetry, spinal deformities, and other congenital malformations of the skull and face bone were observed in $2.6 \%$. The incidence of congenital foot deformities including metatarsus adductus and talipes calcaneovalgus was 2.4\%. Our study suggests that hip dysplasia is the most common musculoskeletal anomaly in CMT, and that the risk of plagiocephaly, skull and facial asymmetry, and spinal and foot deformities is also increased. Among children with CMT, 13.6\% had one or more congenital musculoskeletal deformities. This suggests that CMT can often occur in the complex syndrome of the musculoskeletal system with various congenital deformities.

We compared the neurodevelopmental outcomes of children with CMT who received physical therapy versus those who did 
Table 4. Outcomes of the 5th-7th developmental screening sessions in children with congenital muscular torticollis

\begin{tabular}{|c|c|c|c|c|c|}
\hline Developmental area ${ }^{a)}$ & $\begin{array}{l}\text { Nontreatment group } \\
\qquad(\mathrm{n}=914)\end{array}$ & $\begin{array}{l}\text { Treatment group } \\
\qquad(\mathrm{n}=805)\end{array}$ & $P$ value & RR (95\% Cl) & $\begin{array}{l}\text { Adjusted RR }{ }^{\mathrm{b})} \\
(95 \% \mathrm{Cl})\end{array}$ \\
\hline Total & & & 0.10 & $0.98(0.95-1.01)$ & $0.97(0.93-0.99)$ \\
\hline Appropriate & $706(94.4)$ & $643(96.3)$ & & & \\
\hline Need for evaluation ${ }^{c)}$ & $42(5.6)$ & $25(3.7)$ & & & \\
\hline Communication & & & 0.37 & $0.71(0.31-1.63)$ & $0.41(0.14-1.24)$ \\
\hline Appropriate & 741 (99.6) & 674 (99.9) & & & \\
\hline Need for follow-up & $3(0.4)$ & $1(0.2)$ & & & \\
\hline Gross motor & & & 0.56 & $0.70(0.30-1.61)$ & $0.56(0.20-1.58)$ \\
\hline Appropriate & $730(98.1)$ & $665(98.5)$ & & & \\
\hline Need for follow-up & $14(1.9)$ & $10(1.5)$ & & & \\
\hline Fine motor & & & 0.92 & $1.01(0.48-2.10)$ & $0.77(0.26-1.56)$ \\
\hline Appropriate & 728 (97.9) & 661 (97.9) & & & \\
\hline Need for follow-up & $16(2.2)$ & $14(2.1)$ & & & \\
\hline Problem-solving & & & 0.79 & $0.88(0.42-1.82)$ & $0.58(0.21-1.56)$ \\
\hline Appropriate & $730(97.7)$ & 661 (97.9) & & & \\
\hline Need for follow-up & $17(2.3)$ & $14(2.1)$ & & & \\
\hline Personal/social & & & 0.53 & $0.68(0.33-1.39)$ & $0.57(0.21-1.51)$ \\
\hline Appropriate & $729(97.6)$ & $662(98.1)$ & & & \\
\hline Need for follow-up & $18(2.4)$ & $13(1.9)$ & & & \\
\hline
\end{tabular}

Values are presented as number (\%).

$\mathrm{RR}$, risk ratio; Cl, confidence interval; K-ASQ, Korean Ages and Stages Questionnaire.

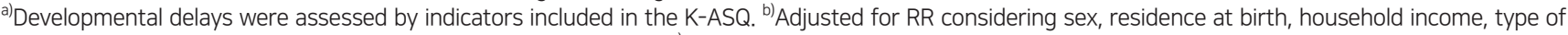
milk feeding, prematurity, birth weight, as recorded in the database. ")"Need for evaluation" was defined by the results of "need for follow-up," "recommendation for further evaluation," and "need for consistent management."

not. In the total assessment, the risk of neurodevelopmental delay was higher in the untreated children than in the treated children. However, there was no significant difference between the 2 groups in each developmental area.

Several studies have shown that the risk of neurodevelop. mental delays increases in children with CMT. Öhman et al. ${ }^{11)}$ studied 82 infants with CMT between 2 and 18 months of age and found an increased risk of delay in early motor milestones until 10 months of age. Schertz et al. ${ }^{12)}$ examined neurodevelopmental outcomes based on telephone interviews for children who were aged 7-9 years and had a history of CMT, and they found developmental delays/disorders in 30 of 68 (44.1\%) of the children. They suggested that the risk of neurodevelopmental disorder such as developmental coordination disorder (32\%), and attention deficit hyperactivity disorder (44\%) increased in children with CMT. Some studies have also reported contradictory outcomes. In a recent study of children aged 3.5-5 years, patients diagnosed with CMT in infancy were not at a higher risk of delayed motor development at preschool age. ${ }^{31)}$ Although the mechanism underlying the increased risk of delayed motor development and neurodevelopmental disorders in children with CMT remains unclear, various hypotheses have been proposed. Hylton ${ }^{21)}$ reported that postural torticollis or tone dysfunction may affect antigravity postural control or balance. Michel and Harkins ${ }^{32)}$ reported that complex postural asymmetry affects the development of motor preferences, as shown by the development of dextral bias in handedness development. In addition, in children with CMT, asymmetry or absence of fidgety movements in early infancy may present other variants of abnormal general movement or provide an important factor that increases the risk of later neurodevelopmental disorders. ${ }^{12)}$

There is evidence that early physical therapies for neckstretching improve neurodevelopmental outcomes in children with CMT. In one study that followed up the gross motor and cognitive development of 101 children with CMT at a mean age of 2.9 months, the risk of a delay in early gross motor development was increased; however, physical therapy normalized most of the children by 10-12 months of age. ${ }^{9)}$

In our study, physical therapy for CMT was found to be effective in overall development, but the effect of development in individual areas is unclear. We assumed that this is not because physical therapy is insufficient. Rather, this may be due to most infants with CMT appearing to have an appropriate outcome in communication, problem-solving, and personal/ social developmental area. Although this study did not show clear evidence that the motor domains of ASQ were improved in patients with CMT after neck-stretching therapy, it would be important to implement of early rehabilitation treatment, as it showed an overall improvement in neurodevelopment after physical therapy.

Early physical therapy with neck-stretching is more effective than later therapy in children with CMT. Infants who started early intervention within 6 weeks of age showed better outcomes than those in whom early intervention was initiated 6 weeks later. ${ }^{16)}$ One study reported that if treatment is started 
by 1 month of age, 98\% of infants are normalized within 1.5 months; however, if treatment is not started by 1 month of age, physical therapy is prolonged for up to 6 months. Furthermore, if treatment is not started by 6 months of age, then it can be required for 9-10 months. ${ }^{33)}$ Demirbilek and Atayurt ${ }^{34)}$ reported that surgery was not required for infants who started physical therapy before 3 months of age; however, $25 \%$ of those who started treatment after 3 months of age required surgery.

In our study, $83.7 \%$ of the treatment group received physical therapy within the first 6 months of life, and the remaining $16.3 \%$ received between $6-12$ months of age. Because these children did not receive any further physical therapy after 1 year of age, we can assume that most children with CMT who do not have comorbidities are presumed to have good outcomes.

The strength of our study is that it is a large-scale study based on comprehensive and nationwide data of children with CMT. In addition, to our knowledge, this is the first study to analyze the incidence of comorbidities and neurodevelopmental outcomes for children with CMT who were born in South Korea.

However, this study also has some limitations. We have only performed a retrospective analysis of data recorded using ICD codes. Therefore, we may not have derived detailed and accurate results of comorbid disorders and diseases in children with CMT. We did not include positional torticollis and did not suggest risk factors of CMT individually such as fetal position in the uterus and difficult delivery. Furthermore, we have not been able to complete long-term follow-up on the neurodevelopmental outcomes of children with torticollis. Therefore, detailed studies on long-term follow-up of neurodevelopmental outcomes in children with CMT will be needed.

In conclusion, if CMT is suspected, early diagnosis should be established, and comorbidities should be identified. In addition, active early treatment should be performed to prevent neurodevelopmental delays and associated musculoskeletal disorders and to promote normal growth and developmental outcomes.

\section{Footnotes}

Conflicts of interest: No potential conflict of interest relevant to this article was reported.

Funding: This study received no specific grant from any funding agency in the public, commercial, or not-for-profit sectors.

Acknowledgments: The authors thank the National Health Insurance Service.

ORCID:

Kyu Young Chae @ https://orcid.org/0000-0003-3243-5853

Man Yong Han @ https://orcid.org/0000-0002-9077-5779

\section{References}

1. Oledzka MM, Sweeney JK, Evans-Rogers DL, Coulter C, Kaplan SL. Experiences of parents of infants diagnosed with mild or severe grades of congenital muscular torticollis. Pediatr Phys Ther 2020;32:322-9.

2. Carenzio G, Carlisi E, Morani I, Tinelli C, Barak M, Bejor M, et al. Early rehabilitation treatment in newborns with congenital muscular torticollis. Eur J Phys Rehabil Med 2015;51:539-45.

3. Tomczak KK, Rosman NP. Torticollis. J Child Neurol 2013;28:365-78.

4. Do TT. Congenital muscular torticollis: current concepts and review of treatment. Curr Opin Pediatr 2006;18:26-9.

5. Wei JL, Schwartz KM, Weaver AL, Orvidas LJ. Pseudotumor of infancy and congenital muscular torticollis: 170 cases. Laryngoscope 2001;111: 688-95.

6. Hussein MA, Yun IS, Lee DW, Park H, Oock KY. Cervical spine dysmorphism in congenital muscular torticollis. J Craniofac Surg 2018;29:925-9.

7. Cheng J, Tang S, Chen T, Wong M, Wong E. The clinical presentation and outcome of treatment of congenital muscular torticollis in infants-a study of 1,086 cases. J Pediatr Surg 2000;35:1091-6.

8. Minihane KP, Grayhack JJ, Simmons TD, Seshadri R, Wysocki RW, Sarwark JF. Developmental dysplasia of the hip in infants with congenital muscular torticollis. Am J Orthop (Belle Mead NJ) 2008;37:E155-8.

9. Schertz M, Zuk L, Zin S, Nadam L, Schwartz D, Bienkowski RS. Motor and cognitive development at one-year follow-up in infants with torticollis. Early Hum Dev 2008;84:9-14.

10. Xiao Y, Chi Z, Yuan F, Zhu D, Ouyang X, Xu W, et al. Effectiveness and safety of massage in the treatment of the congenital muscular torticollis: a systematic review and meta-analysis protocol. Medicine (Baltimore) 2020;99:e21879.

11. Öhman A, Nilsson S, Lagerkvist AL, Beckung E. Are infants with torticollis at risk of a delay in early motor milestones compared with a control group of healthy infants? Dev Med Child Neurol 2009;51:545-50.

12. Schertz M, Zuk L, Green D. Long-term neurodevelopmental follow-up of children with congenital muscular torticollis. J Child Neurol 2013; 28:1215-21.

13. Zhou X, Du Q. High risk of motor development delay in children with congenital muscular torticollis. Ann Phys Rehabil Med 2018;61:e299.

14. Amaral DM, Cadilha RP, Rocha JAG, Silva AIG, Parada F. Congenital muscular torticollis: where are we today? A retrospective analysis at a tertiary hospital. Porto Biomed J 2019;4:e36.

15. Kaplan SL, Coulter C, Fetters L. Physical therapy management of congenital muscular torticollis: an evidence-based clinical practice guideline from the section on pediatrics of the American Physical Therapy Association. Pediatr Phys Ther 2013;25:348-94.

16. Lee $\mathrm{K}$, Chung E, Lee BH. A comparison of outcomes of asymmetry in infants with congenital muscular torticollis according to age upon starting treatment. J Phys Ther Sci 2017;29:543-7.

17. Cheng JC, Wong MW, Tang SP, Chen TM, Shum SL, Wong EM. Clinical determinants of the outcome of manual stretching in the treatment of congenital muscular torticollis in infants: a prospective study of eight hundred and twenty-one cases. J Bone Joint Surg Am 2001;83:679-87.

18. Moon JS. Review of National Health Screening Program for infant and children in Korea. J Korean Med Assoc 2010;53:377-85.

19. Kim JH, Lee JE, Shim SM, Ha EK, Yon DK, Kim OH, et al. Cohort profile: National Investigation of Birth Cohort in Korea study 2008 (NICKs-2008). Clin Exp Pediatr 2021;64:480-8.

20. Stellwagen L, Hubbard E, Chambers C, Jones KL. Torticollis, facial asymmetry and plagiocephaly in normal newborns. Arch Dis Child 2008; 93:827-31.

21. Hylton N. Infants with torticollis: the relationship between asymmetric head and neck positioning and postural development. Phys Occup Ther Pediatr 1997;17:91-117.

22. Boere-Boonekamp MM, van der Linden-Kuiper LT. Positional preference: prevalence in infants and follow-up after two years. Pediatrics 2001; 107:339-43.

23. Ho B, Lee E, Singh K. Epidemiology, presentation and management of 
congenital muscular torticollis. Singapore Med J 1999;40:675-9.

24. Davids J, Wenger D, Mubarak S. Congenital muscular torticollis: sequela of intrauterine or perinatal compartment syndrome. J Pediatr Orthop 1993;13:141-7.

25. Hardgrib N, Rahbek O, Møller-Madsen B, Maimburg R. Do obstetric risk factors truly influence the etiopathogenesis of congenital muscular torticollis? J Orthop Traumatol 2017;18:359-64.

26. Chen HX, Tang SP, Gao FT, Xu JL, Jiang XP, Cao J, et al. Fibrosis, adipogenesis, and muscle atrophy in congenital muscular torticollis. Medicine (Baltimore) 2014;93:e138.

27. Lee SJ, Han JD, Lee HB, Hwang JH, Kim SY, Park MC, et al. Comparison of clinical severity of congenital muscular torticollis based on the method of child birth. Ann Rehabil Med 2011;35:641-7.

28. Li D, Wang K, Zhang W, Wang J. Expression of Bax/Bcl-2, TGF- $\beta 1$, and type III collagen fiber in congenital muscular torticollis. Med Sci Monit 2018;24:7869-74.

29. Hussein MA, Yun IS, Park H, Kim YO. Cervical spine deformity in longstanding, untreated congenital muscular torticollis. J Craniofac Surg 2017;28:46-50.

30. Cheng JC, Au AW. Infantile torticollis: a review of 624 cases. J Pediatr Orthop 1994;14:802-8.
31. Öhman A, Beckung E. Children who had congenital torticollis as infants are not at higher risk for a delay in motor development at preschool age. PMR 2013;5:850-5.

32. Michel GF, Harkins DA. Postural and lateral asymmetries in the ontogeny of handedness during infancy. Dev Psychobiol 1986;19:247-58.

33. Petronic I, Brdar R, Cirovic D, Nikolic D, Lukac M, Janic D, et al. Congenital muscular torticollis in children: distribution, treatment duration and out come. Eur J Phys Rehabil Med 2009;46:153-7.

34. Demirbilek S, Atayurt HF. Congenital muscular torticollis and sternomastoid tumor: results of nonoperative treatment. J Pediatr Surg 1999; 34:549-51.

How to cite this article: Kim OH, Lee SW, Ha EK, Kim JH, Jo YH, Rhie S, et al. Neurodevelopmental outcomes and comorbidities of children with congenital muscular torticollis: evaluation using the National Health Screening Program for Infants and Children database. Clin Exp Pediatr 2022;65:312-9. https://doi.org/10.3345/cep.2021.01417 